\title{
A PEDICLED MUSCLE FLAP BASED SOLELY ON A NEURAL PEDICLE
}

\author{
GULDEN AVCI, M.D., ${ }^{1 *}$ MITHAT AKAN, M.D., ${ }^{2}$ TAYFUN AKOZ, M.D., ${ }^{2}$ WILLIAM KUZON, M.D., ${ }^{3}$ and AYLIN EGE GUL, M.D. ${ }^{4}$
}

\begin{abstract}
We tested the hypothesis that the intrinsic vascular plexus of the motor nerve could support viability in a rat hindlimb muscle flap. In a preliminary study, we examined the course and vascularity of the sciatic nerve, the peroneal nerve, and the peroneous longus muscle in the rat hindlimb via anatomic dissection, microangiography, and histologic study ( $n=10$ animals). On the basis of this examination, the peroneous longus muscle was chosen as our experimental model in this study. In 12 animals, the peroneus longus was acutely elevated, which severed all tendinous and vascular structures, this left the muscle pedicled on the motor nerve only (Group I). Animals in Group II underwent a staged elevation of the flap with division of the vascular pedicle, the tendon of insertion, and the tendon of origin during separate procedures that were 5 days apart $(n=12)$. Muscle viability was evaluated by gross inspection, measurement of muscle weight and length, nitroblue tetrazlium (NBT) staining, microangiography, and histology. NBT staining demonstrated that immediate elevation of the peroneus longus muscle flaps led to an average necrotic area of $80.6 \% \pm 9.8 \%$ (Group I). A significant improvement in viability was observed for muscle flaps of animals in Group II, with peroneus longus muscle necrosis averaging $25.6 \% \pm 9.3 \%$. Microangiography demonstrated that the intrinsic vascularity of nerve was increased dramatically in Group II. These data support the hypothesis that the intrinsic vascular plexus of the motor nerve of a skeletal muscle can support at least partial viability of a muscle flap. However, this vascular axis is inadequate to support complete viability of a muscle flap if the flap is elevated immediately. If a staged elevation affects a surgical delay, the viability of a muscle flap elevated on a neural pedicle can be increased significantly. With adjustments in the delay procedure, this strategy may allow transfer of muscle flaps when maintenance or reconstitution of the primary vascular axis is not possible. (c) 2009 Wiley-Liss, Inc. Microsurgery 29:218-225, 2009.
\end{abstract}

Anatomic studies have demonstrated that peripheral nerves are abundantly vascularized throughout their length by a succession of longitudinal vessels that repeatedly divide and anastomose. ${ }^{1}$ This longitudinal blood supply is exploited in vascularized nerve grafts, where necrosis of the central core of a large nerve trunk can be prevented by preserving the axial vascularity. Masquelet et al. introduced the concept that skin island flaps can be supplied by the vascular axis of superficial, cutaneous nerves in the leg. ${ }^{2}$ On the basis of this work, Akyurek et al. introduced the neural island flap, which is supplied solely by blood from the intrinsic vascular plexus of a cutaneous nerve. ${ }^{3}$ These studies demonstrated that properly designed skin islands can be transferred based on the intrinsic vascularity of a peripheral nerve.

We hypothesized that, like skin, skeletal muscles could be carried as vascularized flaps on the vascular plexus intrinsic to their motor nerve. We designed a neural-pedicled flap on the peroneus longus muscle in the rat hindlimb and tested our hypothesis in flaps elevated immediately following or some time after a staged surgical delay.

${ }^{1}$ Department of Plastic and Reconstructive Surgery, Canakkale Onsekiz Mart University Faculty of Medicine, Canakkale, Turkey

${ }^{2}$ Department of Plastic and Reconstructive Surgery, Dr. Lutfi Kirdar Kartal Education and Research Hospital, Istanbul, Turkey

${ }^{3}$ Department of Plastic and Reconstructive Surgery, University of Michigan, Ann Arbor, Ml

${ }^{4}$ Department of Pathology, Dr. Lutfi Kirdar Kartal Education and Research Hospital, Istanbul, Turkey

${ }^{*}$ Correspondence to: Gulden Avci, M.D., Yeni Kordon Soydan 3, Apt. No. 75 Barbaros, Canakkale, Turkey. E-mail: drguldenavci@hotmail.com

Received 22 July 2008; Accepted 24 October 2008

Published online 9 February 2009 in Wiley InterScience (www.interscience.wiley. com). DOI 10.1002/micr.20609

\section{MATERIALS AND METHODS}

A total of 34 Wistar rats of both sexes, weighing 200-250 g, were used in this experiment. The rats were housed in individual cages within a temperature- and light-controlled room. All animals were given the same commercial food and tap water. Study approval and consent were obtained from our institutional Ethical Review Committee. We used only left legs of all rats.

\section{Anatomic Study}

Ten rats were sacrificed by an overdose of ether inhalation. An incision was made from sacrum to lateral malleol (Fig. 1a). An anatomic dissection was done to determine the anatomy of the sciatic and peroneal nerves and their relationship to the peroneus longus muscle. In the popliteal fossa, the sciatic nerve splits in to three parts: sural, peroneal, and tibial nerve (Fig. 1b). The peroneal nerve courses parallel to the posterior surface of the peroneus longus muscle and splits into two branches (Fig. 1c). The diameter of the nerve measured $1 \mathrm{~mm}$ in the popliteal fossa. On the basis of the intrinsic vascularity of the peroneal nerve, the elevation of the peroneus longus muscle flap was planned because of the proximity of the large diameter nerve to the muscle.

Microangioraphy was performed to visualize the vascularity of the peroneal nerve and the peroneus longus muscle. Microangiography was performed as described elsewhere. $^{4,5}$ Briefly, after cannulation of the femoral artery of the recipient site, a $30 \%$ solution of finely divided barium sulfate (Micropaque) was injected with a syringe, with $20-25 \mathrm{~mL}$ of the solution. The muscle flaps 


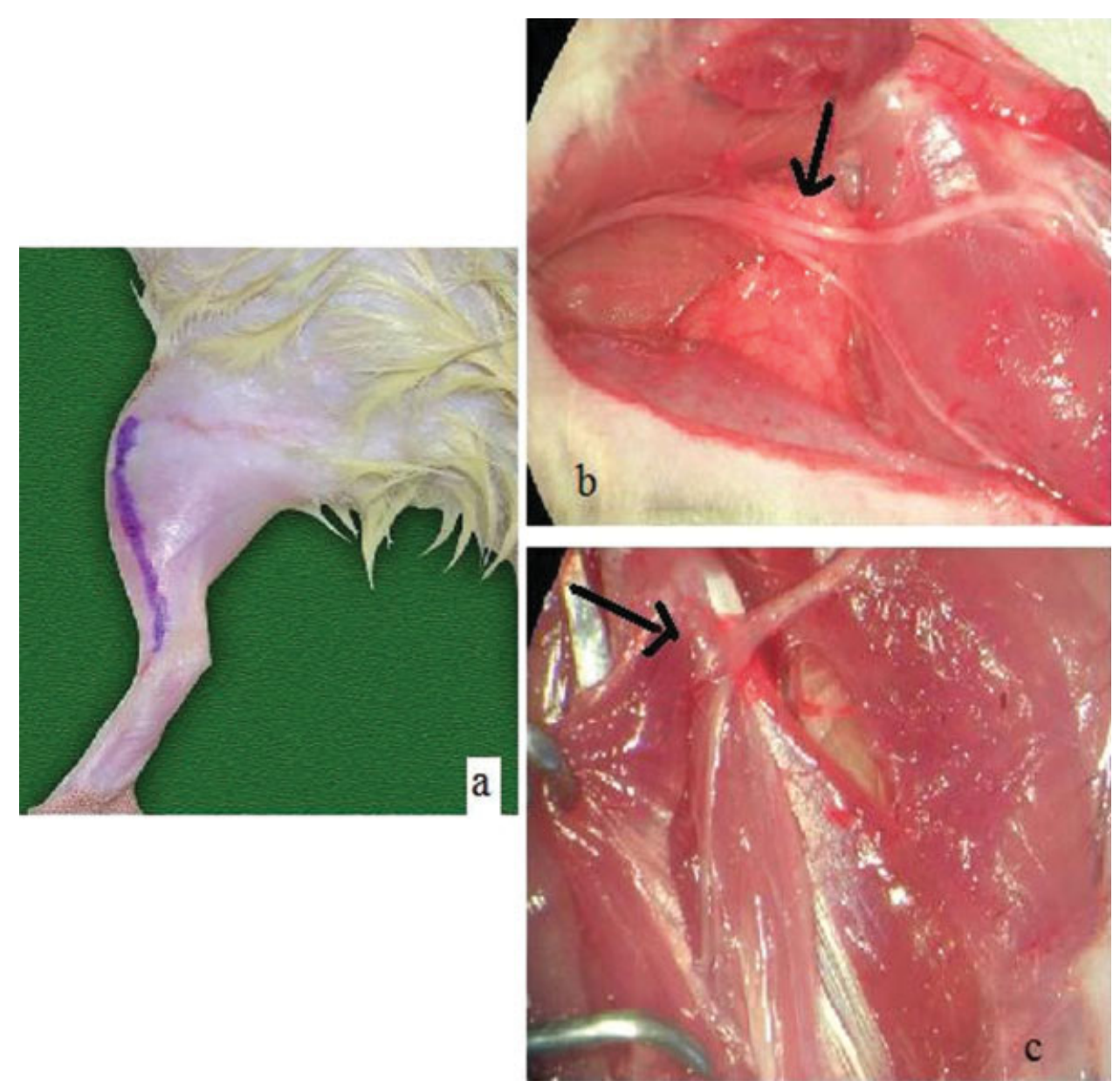

Figure 1. (a) View of the incision made over the lower extremity. (b) The three divisions of Siatic nerve in the poplitea are identified by the arrow. (c) The two divisions of the peroneal nerve below the muscle is identified by the arrow. [Color figure can be viewed in the online issue, which is available at www.interscience.wiley.com.]

with neural pedicule were stored in a refrigerator at $4^{\circ} \mathrm{C}$ for 4 hours. All flaps then underwent radiography with a soft X-ray machine (Mammo Diagnost UC, Philips, Hamburg, Germany) at setting of $24 \mathrm{kV}$ and $10 \mathrm{Ma}$ (see Fig. 2). The weight and length of the peroneus longus muscle were measured, and the muscle and peroneal nerve were harvested for histological study (H\&E, 40×).

\section{Creation of an Island Neural Pedicle Muscle Flap}

Group I: Immediate neural-pedicled muscle flap. Twelve rats were anesthetized with intramuscular injection of ketamine hydrochloride. An incision was made over the posterolateral aspect of the cruris to expose the peroneus longus muscle. The muscle was dissected from its distal aspect to its proximal aspect, which severed the tendons of origin and insertion. Careful consideration was given to the association of muscle with nerve, and the nerve pedicle of the muscle flap was preserved throughout the dissection. The vascular pedicle was ligated and cut; the nerve pedicle was $3 \mathrm{~mm}$ in width and 5-7 $\mathrm{mm}$

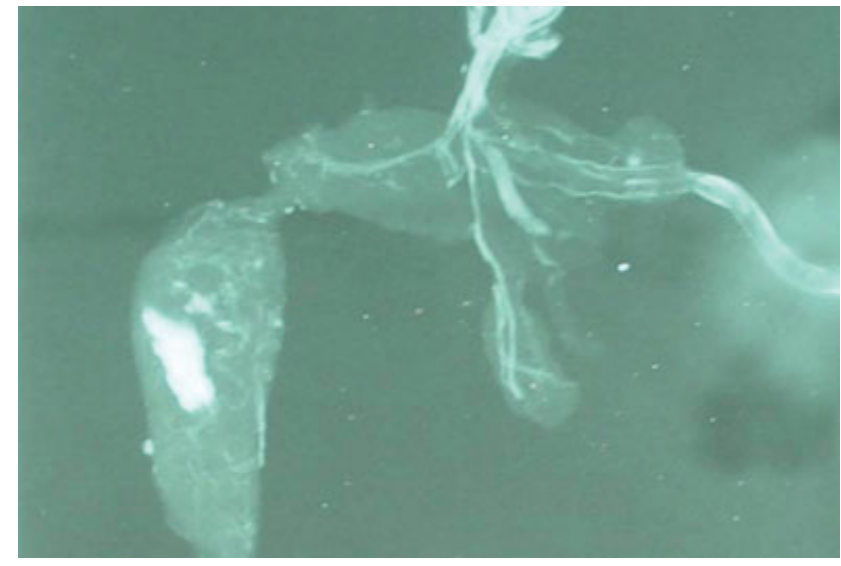

Figure 2. Microangiographic appearance of the normal (control) group's muscle and nerve. [Color figure can be viewed in the online issue, which is available at www.interscience.wiley.com.]

in length (see Fig. 3). After elevating the nerve-pedicled muscle flap, the origin and insertion were repaired with $5 / 0$ vicryl. Skin was sutured with $5 / 0$ silk. On the second 
postoperative day, the animals were sacrificed by an overdose of ether inhalation to evaluate muscle viability.

Peroneus longus muscles were assessed grossly and with microangiography and histology (H\&E, $\times 40)$. Microangiography and histology was performed on one animal in each group. The muscle was excised, and its weight and length were measured. The extent of muscle necrosis was estimated using nitroblue tetrazolium dye. ${ }^{6}$ Muscle specimens were obtained and sectioned transversely into $5 \mathrm{~mm}$ thick slice (Fig. 4a). These were incubated at $37^{\circ} \mathrm{C}$ for 30 minutes in a $0.005 \%$ solution of nitroblue tetrazolium (Sigma Chemical Company, St.

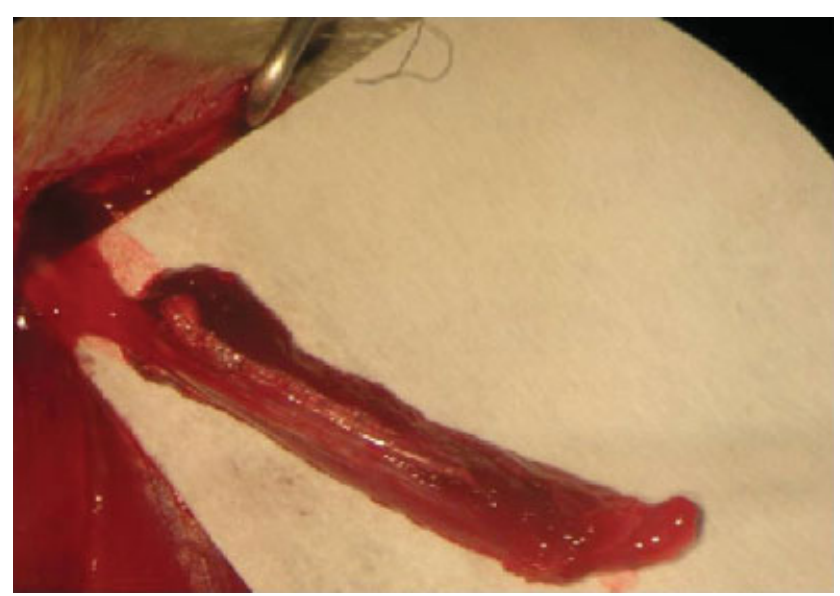

Figure 3. Acutely elevated muscle flap with nerve pedicled. [Color figure can be viewed in the online issue, which is available at www.interscience.wiley.com.]
Louis, MO) in $0.1 M$ phosphate buffer (Fig. 4b). All slices were then examined with regard to color change to determine the viability of the muscle. Dark stained areas were estimated as areas of viable muscle, whereas areas of nonviable muscle remained unstained due to failure to reduce the tetrazolium dye. Viable areas of muscle that were stained dark were traced on acetate sheets. The paper template technique was used to evaluate the percentage of viable muscle.

\section{Group II: Delayed creation of an island muscle}

flap based on a neural pedicle. Twelve rats in this group underwent three procedures to elevate the neuralpedicled muscle flap, affecting a staged surgical delay. In the first procedure, the vascular pedicle of the peroneus longus muscle was ligated and cut; the origin and insertion of muscle were protected. Special attention was paid to maintain the intimate relation of muscle and, the muscle was undermined and so a bipedicle flap was created (Fig. 5a). On postoperative day 5, a second delay procedure was performed by dividing the insertion and one third of the peroneus longus muscle origin (Fig. 5b). Ten days after the first operation, a third delayed procedure was performed. In this operation, the origin of the muscle was completely interrupted (Fig. 5c). Two days after the third procedure the rats were sacrificed, and the muscle flaps were evaluated as in the previous group.

Statistical analysis. The GraphPad Prisma V.3 statistical program was used to analyze all the data. Numerical data, including muscle weight and length and the extent of muscle necrosis, were evaluated using either the Mann-Whitney- $U$ test (two group comparisons) or the
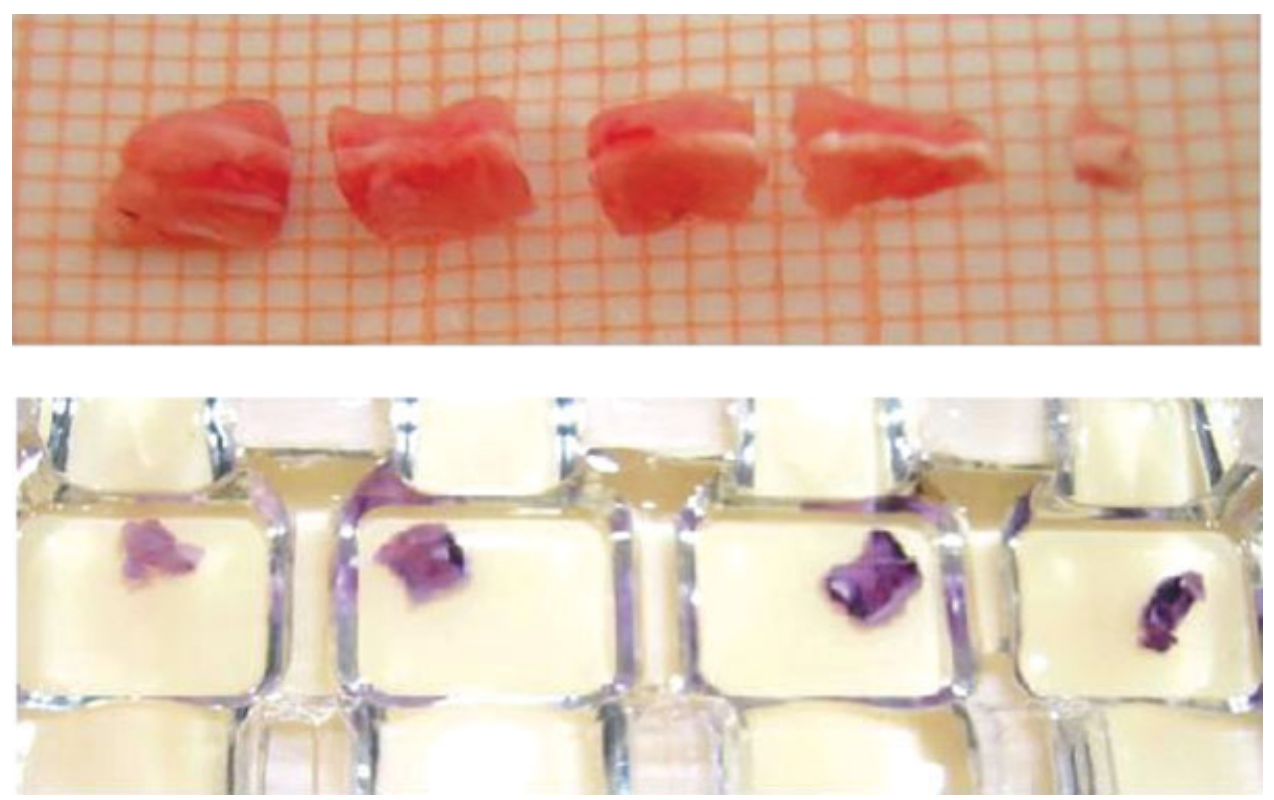

Figure 4. (a) Muscle was sectioned transversely. (b) Slices were incubated in nitroblue tetrazolium solution. [Color figure can be viewed in the online issue, which is available at www.interscience.wiley.com.] 

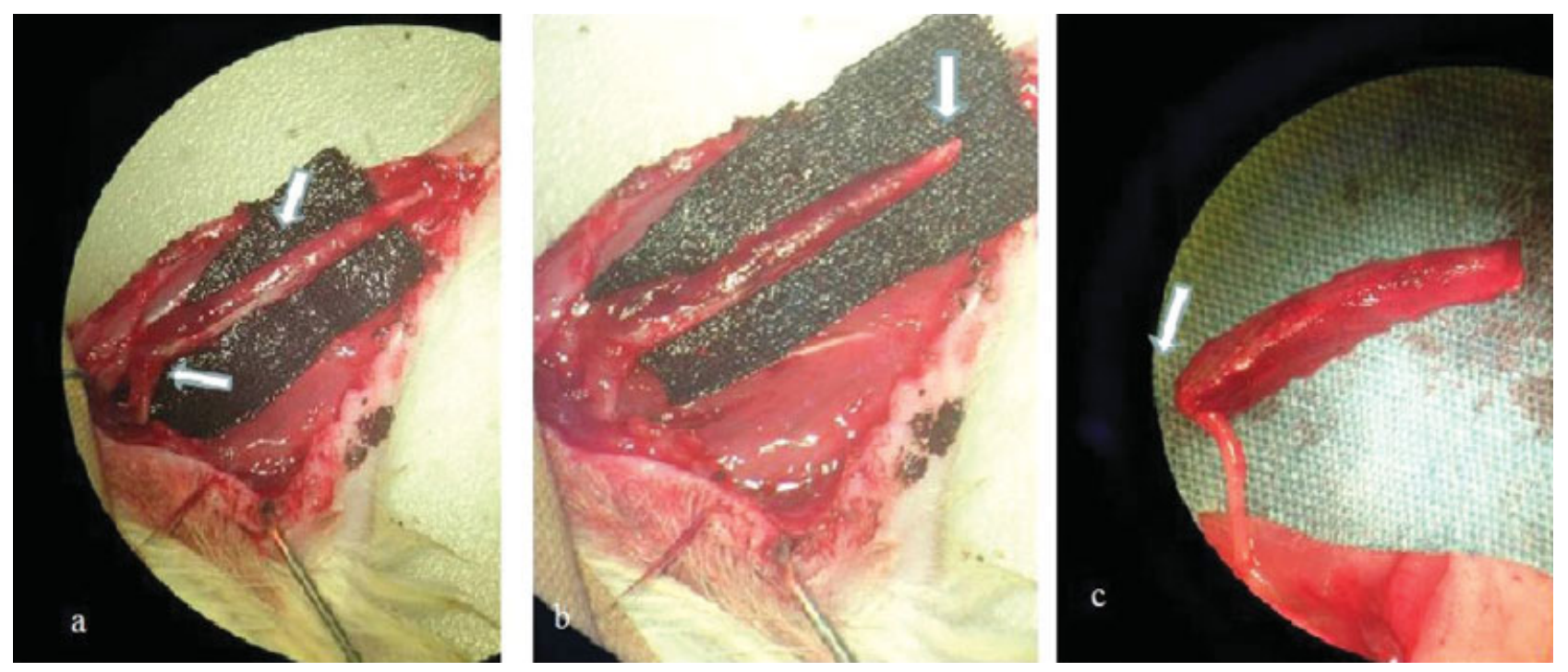

Figure 5. (a) First operation of delayed group, muscle were dissected from ground, origin, and insertion of the muscle were protected (first delay procedure). Vascular pedicles of the muscle were ligated and divided. (b) Second delay procedures, on postoperative day 5 . The insertion of the muscle and1/3 of the origin of muscle was cut and muscle dissected from the ground again. (c) Third delay procedures, on postoperative day 10: whole of the origin of the muscle was interrupted and flap was elevated completely. [Color figure can be viewed in the online issue, which is available at www.interscience.wiley.com.]

Table 1. Statistical Results of the Muscle Weight, Length, and Necrosis Percentage

\begin{tabular}{|c|c|c|c|c|c|}
\hline & Normal muscle $n=10$ & Acutely elevated flap $n=12$ & Delayed flap $n=12$ & & \\
\hline Necrosis (\%) & - & $80.62 \pm 9.83$ & $25.59 \pm 9.32$ & MW:0.0 & $P=0.0003$ \\
\hline Length (mm) & $18.25 \pm 0.71$ & $18.57 \pm 0.79$ & $18 \pm 1.20$ & KW:1.26 & $P=0.53$ \\
\hline Mass (mg) & $1,151 \pm 201.7$ & $1,291 \pm 153.90$ & $1,042 \pm 238.2$ & KW:5.35 & $P=0.06$ \\
\hline
\end{tabular}

MW, Mann-Withney U test; KW, Kruskal-Wallis test.

Kruskal-Wallis test (multiple group comparisons). The level of statistical significance chosen was a $P$-value of less than 0.05. Numerical results are presented as mean \pm standard deviation.

\section{RESULTS}

\section{Characteristics of the Peroneus Longus Neural Muscle Flap}

The mean peroneus longus muscle weight was $1,151 \pm$ $201.7 \mathrm{mg}$, and the mean length of muscle was $18.25 \pm$ $0.71 \mathrm{~mm}$ (Table 1). Upon gross examination, the muscle belly comprised the proximal two thirds of the total length, from origin to insertion; the distal third of this length was composed of aponeurosis and tendon. Microscopic examination of nerve and muscle showed that the nerve had an abundant longitudinal intrinsic vascular plexus.

\section{Neural-Muscle Flap Investigation}

Direct inspection. In flaps elevated immediately, most of the muscle was pale, had considerable edema, and was fragile. Only the proximal segment of the muscle appeared viable (Fig. 6a). The mean weight of the muscle was $1,291 \pm 153.90 \mathrm{mg}$; the mean length was $18.57 \pm$ $0.79 \mathrm{~mm}$. In flaps elevated after a staged delay, the proximal three fifths of the muscle appeared viable, with the remaining distal portion of the muscle appearing pale and nonvascularized (Fig. 6b). Increased vascularity and a thickened adventitia were observed in the extramuscular portion of the motor nerve for delayed flaps. A similar increased vascularity was observed for the intramuscular portion of the motor nerve. The mean weight of the muscle was $1,042 \pm 238.2 \mathrm{mg}$; the mean length of the muscle was $18 \pm 1.20 \mathrm{~mm}$ (Table 1 ).

Microangiography. In acutely elevated flaps, microangiography demonstrated that the proximal one fifth of the peroneus longus muscle was vascularized; the vascularity of the motor nerve was not different from the anatomic study (Fig. 7a). In the delayed flaps, we observed an increase in the vascularity of the motor nerve. The proximal three fifths of the muscle had an increase in the number of vessels observed (Fig. 7b).

Nitroblue tetrazolium dye stain test. In the acute flaps, the extent of the muscle necrosis was $80.62 \% \pm$ $9.83 \%$ (Table 1). The nitroblue tetrazlium (NBT) staining pattern of the muscle sections was very consistent among 

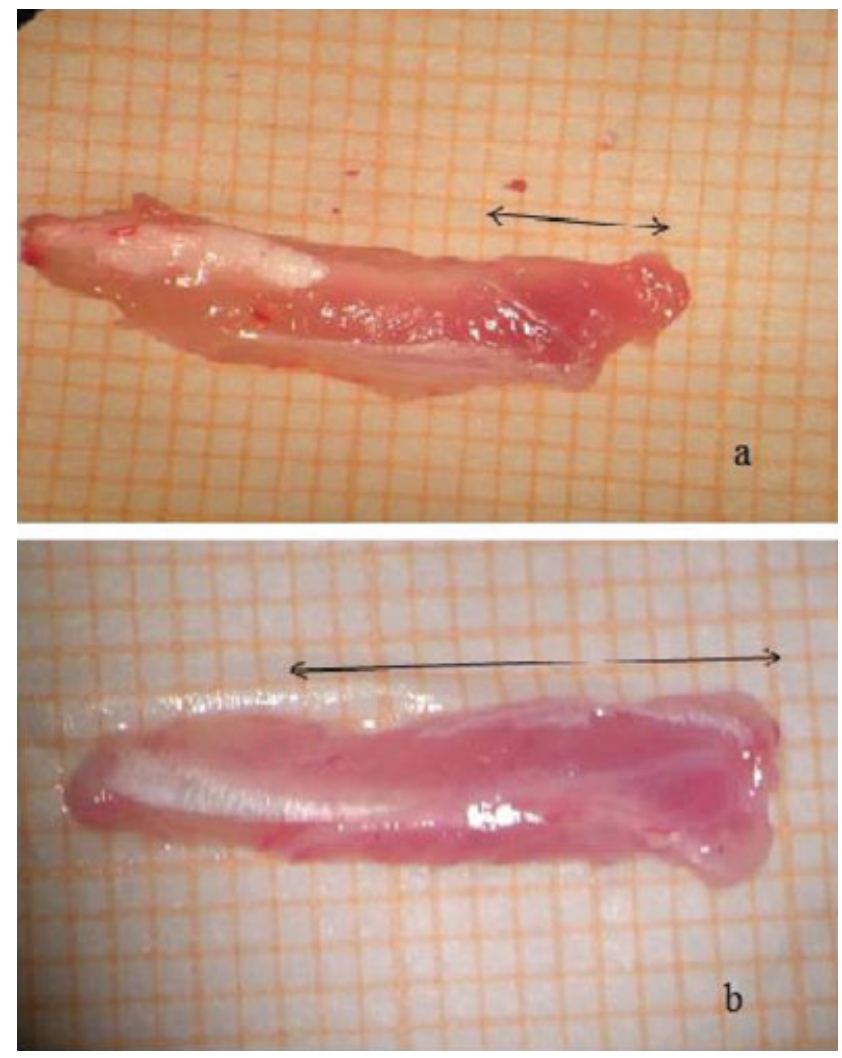

Figure 6. (a) The macroscopic view of the muscle in the acutely elevated muscle flap. The arrow shows that small muscle segment on the proximal are viable. (b) The macroscopic view of the muscle in the delayed group. The arrow shows the length of viable muscle segment. [Color figure can be viewed in the online issue, which is available at www.interscience.wiley.com.]

all the muscles examined. In the proximal one fifth of the muscle, the segments were fully stained, indicating muscle viability. The central areas of the transverse sections from the distal $4 / 5$ of the muscle were unstained, indicating muscle necrosis. Only the peripheral rim of these segments was stained dark blue in color. On the basis of these results, we commented that vessels in tissues that surround the peripheral, surviving rim of the distal segments of the acute flaps were observed. This indicated that these flaps may have survived as muscle grafts with neovascularization arising from the periphery. In the delayed flaps, the percentage of muscle necrosis was $25.59 \pm 9.32 \%$, and again, the staining pattern of the muscle sections was very characteristic (Table 1). The central area of the transverse cut surface of the proximal $3 / 5$ muscle segments was stained, whereas the peripheral areas of these segments were unstained. Segments from the distal $2 / 5$ of the muscle were unstained. There was no rim of surviving muscle in the distal segments of the delayed flap, perhaps because the multiple delay procedures resulted in increased fibrosis on the surface of the
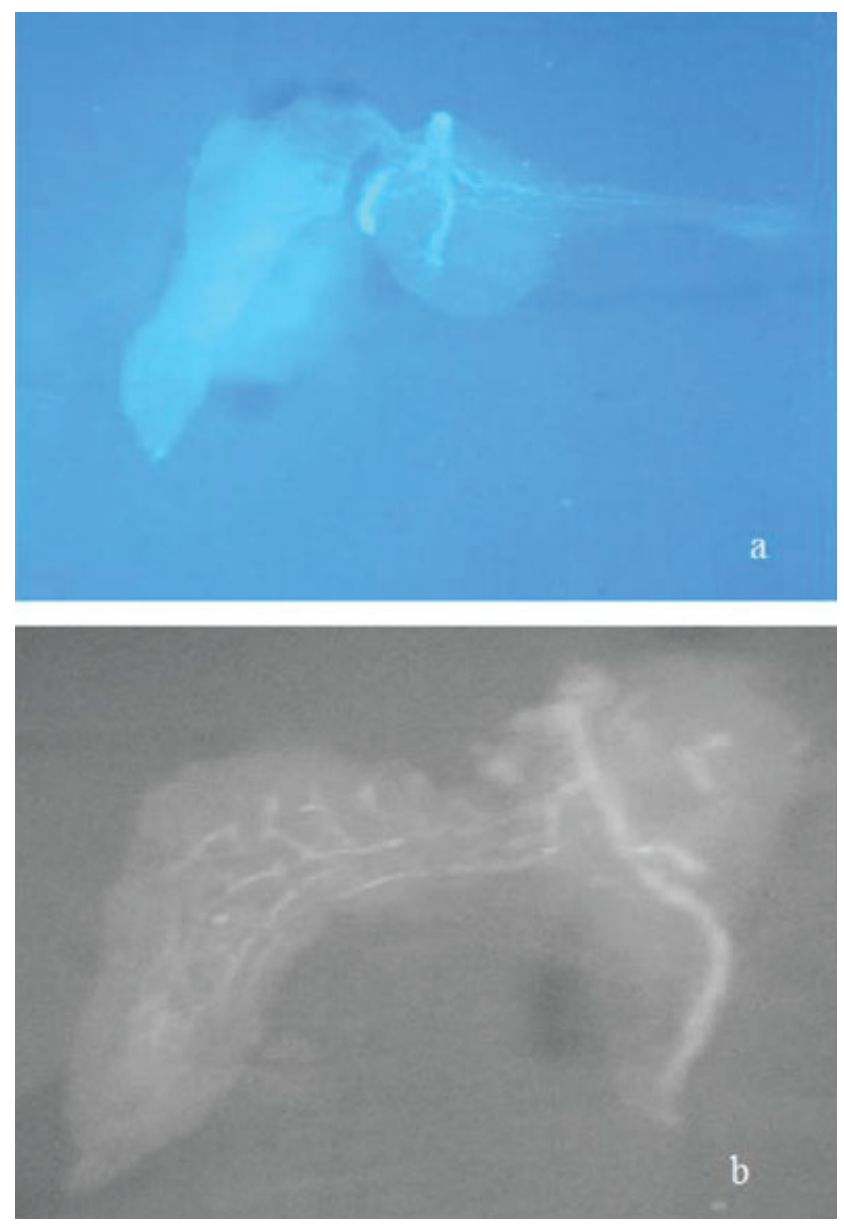

Figure 7. (a) Microangiographic appearance of the acutely elevated muscle flap with nerve pedicled. (b) Microangiographic appearance of the delayed muscle flap with nerve pedicled. [Color figure can be viewed in the online issue, which is available at www.interscience. wiley.com.]

muscles, which prevented peripheral neovascularization in this group.

Histology. In the acute flaps, the necrosis, edema, and invasion of polymorphonuclear leukocytes were seen in the distal portions of muscles that were not viable (Fig. 8a). Edema and degenerative changes were also observed in the motor nerve (Fig. 8c). In the delayed flaps, the majority of the muscle maintained a normal architecture (Fig. 8b), and the number of vascular channels in the nerve was increased significantly (Figs. 8d and $8 \mathrm{e}$ ).

\section{DISCUSSION}

Peripheral nerve trunks are well-vascularized structures. A nerve's blood supply comes from both extrinsic and intrinsic sources. Regional vascular bundles running through adventisa nervorum constitute an "extrinsic vascular system" that reinforces the intraneural, longitudinal- 

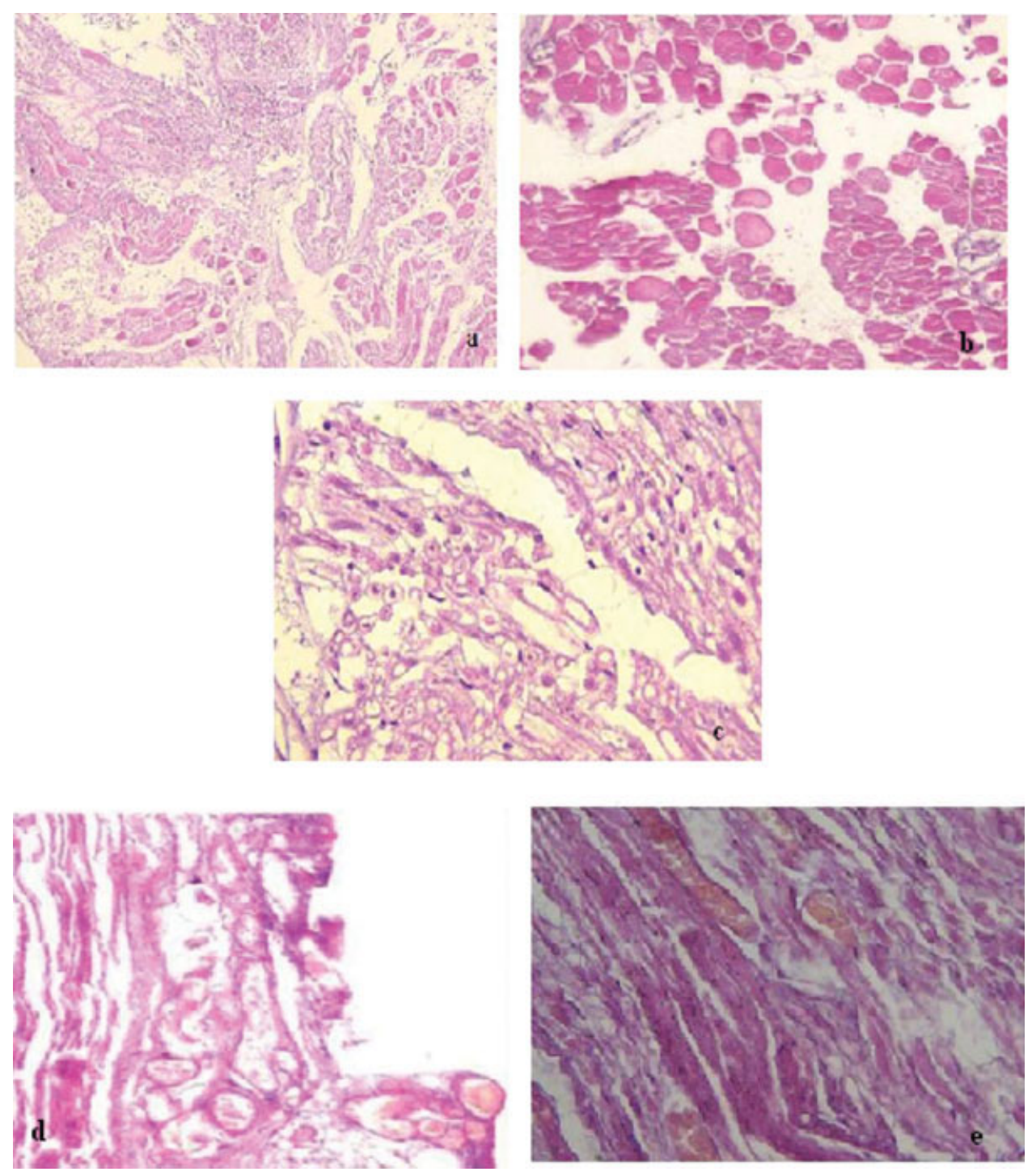

Figure 8. (a) Evaluation of the muscle in acutely elevated flap group histologically $(H \& E, \times 10)$. (b) Evaluation of the muscle in delayed flap group histologically $(H \& E, \times 10)$. (c) Evaluation of the nerve in acutely elevated flap group histologically $(H \& E, \times 40)$. (d,e) Evaluation of the nerve in delayed flap group histologically $(H \& E, \times 40)$. Increment of vascularity of nerve was seen. [Color figure can be viewed in the online issue, which is available at www.interscience.wiley.com.]

oriented microvascular bed. ${ }^{7-10}$ The neurocutaneous flaps' blood supply comes predominantly from the extrinsic vascular system of the nerve. ${ }^{11}$ Akyurek et al. demonstrated the ability to elevate a flap with nerve-intrinsic vascularity. After neural-island flap description, the intrinsic vascularity of the nerve attracted our attention. ${ }^{3}$

When a nerve is elevated for transposition, it is stripped of its extrinsic blood supply and must survive on its intrinsic blood flow. Previous investigations have tried to determine the maximum length of nerve that can be elevated before becoming ischemic. Lundborg et al. found that long nerves could be mobilized, concluding that intraneural microcirculation can resist considerable mobilization. Signs of active microcirculation existed in nerves with a 1:45 diameter-to-length ratio. ${ }^{12}$ Maki et al. surgically mobilized $15 \mathrm{~cm}$ of the rabbit sciatic and tibial nerves and evaluated the effect of mobilization on the capillary blood flow. The nerve was elevated and the epineurium was left intact. The elevation consisted of cutting all extrinsic vessels, motor and sensory branches, and other connective tissues. The blood flow to the nerve was derived only from the intrinsic blood supply. The increased nerve blood flow was significantly higher in the proximal segment, ranging from 2.4 to 4.4 times. When the nerve is mobilized and also transected distally, the blood flow of the nerve is derived only from the proximal intrinsic circulation. Blood flow decreases in the distal segment of the nerve but maintains a diameter-to-length 
ratio up to $1: 63 .{ }^{13}$ Mobilization with nerve transection increases the blood flow. Lundborg noted that many intraneural vessels that are initially closed will open when the nerve is mobilized. He suggested that this could be a protective mechanism against ischemia. ${ }^{10}$ Consequently, we think that the intrinsic vascularity of the nerve can be used as the pedicle of the muscle flap.

Surgical delay may be done to increase vascularity, thereby increasing the length and viability of the flap. Komorowska et al. showed that elevation of a pedicled flap caused significant decrease in distal flap perfusion. Delay was adequate to establish sufficient flap perfusion independent of vascular pedicle. ${ }^{14} \mathrm{~A}$ typical process of flap delay involves raising a rectangular flap on two sides, essentially creating a bipedicle flap. One week later, the 3rd side is divided; division of the third side may also be done in two stages 3 days apart. ${ }^{15}$ Recommendations for the timing of delay vary from several days to several weeks. ${ }^{15-17}$ Pang et al. showed that blood flow in delayed flaps increased within 2 days, reached a plateau after day 3, and showed no change between days 4 and $14 .{ }^{18}$ On the basis of this knowledge we planned our study. Because the muscle flap is very tiny, the flap may be revascularized from the recipient bed if it is harvested for assessment after 3 or more days. A biomaterial sheet could be used to isolate the flap from the bed, but it would cause foreign body reaction. So, we decided to harvest the muscle flap and evaluate its viability at the 2nd postoperative day to avoid revascularization from the recipient bed, which theoretically starts from the 3rd postoperative day. In our study, vascular increase was seen both grossly and histologically after delay procedure. The work by Taylor et al. is important because these authors have demonstrated experimentally that the maximal anatomic effect on the arterial side, secondary to surgical delay, is at the level of the choke vessels that link adjacent vascular territories. Anatomically, these vessels increase appreciably in size in response to a delay. A sequential increase in size of the choke vessel occurs during the delay period, with a rapid increase in size between 48 and 72 hours. ${ }^{19-21}$ We think that open choke vessels can provide muscle viability via the nerve's intrinsic vascular system.

Although the beneficial effects of vascular delay on the survival of skin flaps are widely known, there have been relatively few previous studies on the effect of vascular delay on muscle flaps. Mathes and Vasconez initially demonstrated that the vascular delay procedure significantly increased the survival rate of the dog gracilis muscle from $69 \%$ in nondelayed muscle flaps to $99 \%$ in delayed muscle flaps; ligation of a dominant vascular pedicle was performed 3 weeks before the flap elevation. ${ }^{22}$ Callegari et al. showed that dilation of choke vessels occurred in canine lattissimus dorsi, rectus abdomi- nis, and sartorius muscles after a 3-week delay period. ${ }^{19}$ Baker et al. studied the effects of surgical delay to the microcirculation of the muscle flap. ${ }^{23,24}$ Morris and Taylor have shown that capillary blood flow of the muscle flap increased after the delay procedures performed on the muscle flap. ${ }^{20}$ Morris and Yang showed that delay procedure performed on the dominant vascular pedicle in biceps femoris muscle flap model and the nondominant pedicle in the latissimus dorsi muscle-flap model increases muscle flap capillary blood flow and viability. ${ }^{25}$ Increased blood flow to the muscle flap is secondary to opening and dilating the choke vessels. Various theories try to explain the mechanism of the vascular change that follows the delay of the muscle flap; vasodilatation is secondary to the sempathectomy, hypersensitivity of the arteriovenous shunts, angiogenesis, vascular reorganization, inflammatory mediators and changes in tissue metabolism as factors underlying the delay phenomenon. ${ }^{24}$ Lineaweaver et al. showed that vascular endothelium growth factor could be an important element in the delay phenomenon. ${ }^{26}$ Vascular endothelium growth factor affects microcirculation acutely by change in vascular permeability and in a sustained manner by promoting angiogenesis. ${ }^{26}$ In our study, the muscle flap can survive after the delay based on the intrinsic vascularity of the nerve. Following the delay procedure, muscle atrophy was determined. The possible causes of muscle atrophy may be restricted use of the muscle by the rat and restricted blood flow to the muscle during delay procedures.

Akyurek et al. first described neural-island flap, which is based on intrinsic vascularity of the nerve. The survival rate of the delayed neural-island flaps was found to be $94.5 \%$, depicting a greater survival rate than an acutely-elevated neural-island flap $(38 \%){ }^{3}$ Ozkan et al. harvested the biceps femoris muscle and musculocutaneous flaps based on their motor nerves. Average muscle viability levels of neuromuscular, neuromusculocutaneous, delayed neuromuscular, delayed neuromusculocutaneous, conventional muscle, and musculocutaneous flaps were $20.6 \pm 7.58,22.4 \pm 4.21,86.4 \pm 6.14,85 \pm 4.21,89.6 \pm$ 4.48 , and $88 \pm 5.51 \%$, respectively. ${ }^{27}$ We prefer using the peroneus longus muscle to create muscle flaps based on its motor nerve; the peroneus longus muscle is more distal than the biceps femoris muscle. Managing defects below the knee is more challenging. When the peroneus longus muscle flap with intrinsic vascularity of the nerve was elevated acutely, the percent of muscle viability was found to be $19.38 \%$, and after delay procedures, this increased to $74.41 \%$. We believe that the discrepancy between Akyurek's described viability of delayed skin flap and the viability of our delayed muscle flap is due to the different tissue of the flaps; muscle structure had less resistance to ischemia than skin. Viability of Ozkan 
described delayed muscle flap/musculocutaneous flap is better than viability of our delayed muscle flap. We think this is due to proximality of the biceps femoris muscle; the vascularity increase of biceps femoris muscle flap is greater than the vascularity increase of our peroneus longus muscle flap.

Neural pedicle based flaps have very narrow pedicles, and this pedicle lacks any restriction to a specific pivot point. The muscle flaps are ideal alternatives to manage the complex wounds that traditionally do not respond well to local wound management. Muscle innervation is protected when the muscle flap is elevated based on the intrinsic vascularity of the nerve. We think that muscle functions continue when the muscle flap is elevated nerve based. With this technique, functional muscle transfer may be possible. But we know that further experimental studies must be performed to improve this new concept. We bargain for the development of clinical application of neural pedicle muscle flap. As exemplify, sometimes thoracodorsal vessels are injured during mastectomy, the lattissimus dorsi muscle flap may be elevated based on thoracodorsal nerve. Neural pedicle muscle flap is more easily than free flaps.

\section{CONCLUSION}

Peripheral nerves are well-vascularized structures with a well-developed collateral system. After the intrinsic vascularity of the nerve (which has an extensive blood reserve capacity) is made more functional than normal using delayed surgical procedure, the muscle flap could be elevated using the nerve's intrinsic vascularity. When the tissue mass is added to the nerve as a nerve-based flap, this tissue mass can be nourished by the same vascularity as the nerve.

\section{REFERENCES}

1. Suami H, Taylor GI, Pan WR. Angiosome territories of the nerves of the lower limbs. Plast Reconstr Surg 2003;112:1790-1798.

2. Masquelet AC, Romana MC, Wolf G. Skin island flaps supplied by the vascular axis of the sensitive superficial nerves: Anatomic study and clinical experience in the leg. Plast Reconstr Surg 1992;89:1115-1121.

3. Akyurek M, Safak T, Sonmez E, Ozkan O, Keçik A. A new flap design: Neural- island flap. Plast Reconstr Surg 2004;114:1467-1477.

4. Akyurek M, Safak T, Manavbası I, Keçik A. A rat musculocutaneous flap model: the biceps femoris musculocutaneous flap. Ann Plast Surg 2000;45:305-312.

5. Ozkan O, Akyurek M, Safak T, Kayikcioglu A, Guler G, Erk Y. A new flap model in rats: iliac osteomusculocutaneous flap. Ann Plast Surg 2001;47:161-167.
6. Morris SF, Pang CY, Zhong A, Boyd B, Forrest CR. Assesment of ischemia-induced reperfusion injury in the pig latissimus dorsi myocutaneous flap model. Plast Reconstr Surg 1993;92:1162-1172.

7. Van Beek A, Kleinert HE. Practical microneurorraphy. Ortop Clin North Am 1977;8:377-386.

8. Bell MA, Weddell AG. A morphometric study of intrafascicular vessels of mammalian sciatic nerve. Muscle Nerve 1984;7:524-534.

9. Bell MA, Weddell AG. A descriptive study of the blood vessels of the sciatic nerve in the rat, man and other mammals. Brain 1984; 107:871-898.

10. Lundborg G. Ischemic nerve injury: Experimental studies on intraneural microvascular pathophysiology and nerve function in a limb subjected to temporary circulatory arrest. Scand J Plast Reconstr Surg Hand Surg Supp 1970;6:3-113.

11. Lundborg G. Intraneural microcirculation. Orthop Clin North Am 1988;19:1-12.

12. Lundborg G. The intrinsic vascularization of human peripheral nerves. Structural and functional aspects. J Hand Surg 1979;4:34-41.

13. Maki Y, Firrell JC, Breidenbach W. Blood flow in mobilized nerves: Results in a rabbit sciatic nerve model. Plast Reconstr Surg 1997; 100:627-635.

14. Komorowska-Timek E, Timek TA, Brady S, Buncke H. Perfusion, viability, and pedicle dependence in acute and delayed rat island skin flaps. Microsurg 2007;27:146-151.

15. Smith JD, Pribaz JJ. Flaps. In:Achuer BM,Erikson E, editors. Plastic Surgery: Indications, Operations, and Outcomes. St. Louis: Mosby; 2000. pp 261-292.

16. Chase RA. Introduction: The history of vascularized composite tissue transfer. In: Strauch B, Vasconez LO, Hall-Findlay ED, editors. Grabb's Encyclopedia of Flaps, 2nd ed. Philadelphia: Lippincot-Raven; 1998. p xiii.

17. Milton SH. The effects of delay on the survival of experimental studies on pedicled skin flaps. Br J Plast Surg 1969;22:244-252.

18. Pang CY, Forrest CR, Neligan PC, Lindsay WK. Augmentation of blood flow in delayed random skin flaps in the pig: Effect of length of delay period and angiogenesis. Plast Reconstr Surg 1986;78:6874.

19. Callegari PR, Taylor GI, Caddy CM, Minabe T. An anatomic review of the delay phenomenon. I. Experimental studies Plast Reconstr Surg 1992;89:397-418.

20. Morris SF, Taylor GI. The time sequence of the delay phenomenon: When is a surgical delay effective? An experimental study. Plast Reconstr Surg 1995;95:526-533.

21. Taylor GI, Corlett RJ, Caddy CM, Zelt RG. An anatomic review of the delay phenomenon. II. Clinical applications Plast Reconstr Surg 1992;89:408-418.

22. Mathes SJ, Vasconez LO. Myocutaneous free-flap transfer: Anatomical and experimental considerations. Plast Reconstr Surg 1978; 62:162-166.

23. Boyd JB, Markland B, Dorion D, Pang CY, Morris S. Surgical augmentation of skin blood flow and viability in pig musculocutaneous flap model. Plast Reconstr Surg 1990;86:731-738.

24. Barker JH, van Aalst VC, Keelen PC, Bidiwala S, Frank JM, Carroll SM, Wan C, Carroll CM, Anderson GL, Joels C, Tobin GR. Vascular delay in skeletal muscle: A model for microcirculatory studies. Plast Reconstr Surg 1997;100:665-669.

25. Morris SF, Yang D. Effect of vascular delay on viability, vasculature, and perfusion of muscle flaps in the rabbits. Plast Reconstr Surg 1999; 104:1041-1047.

26. Lineaweaver WC, Lei MP, Mustain W, Oswald TM, Cui D, Zhang F. Vascular endothelium growth factor, surgical delay, and skin flap survival. Ann Surg 2004;239:866-875.

27. Ozkan O, Akyürek M, Safak T, Acar B, Ozgentaş HE, Keçik A. Neuromuscular and neuromusculocutaneous flaps in the rat. J Plast Reconstr Aesthet Surg 2006;59:279-290. 